\title{
Disease Management and Outcomes in Patients Hospitalized for Acute Heart Failure in Japan
}

\author{
Lei Chen · Raluca Ionescu-Ittu • Hela Romdhani · Annie Guerin • \\ Paul Kessler · Maria Borentain · Keith Friend · Mary DeSouza • \\ Naoki Sato
}

Received: November 11, 2020 / Accepted: January 18, 2021 / Published online: February 20, 2021

(c) The Author(s) 2021

\begin{abstract}
Introduction: This study described patients hospitalized for acute heart failure (AHF) in Japan who received intravenous (IV) diuretics and/or vasodilators as the initial therapy.

Methods: The Japan Medical Data Vision database was used to identify adult patients hospitalized for AHF during 2013-2017, who were hemodynamically stable at presentation and treated with IV diuretics and/or IV vasodilators as initial therapy. Treatment patterns and use of cardiac rehabilitation, as well as outcomes (e.g., length of stay [LOS], in-hospital mortality, HFreadmission) were reported overall and by year of AHF hospitalization.
\end{abstract}

Supplementary Information The online version contains supplementary material available at https:// doi.org/10.1007/s40119-021-00212-y.

\section{Chen}

Employee of Bristol Myers Squibb at the time when the research was conducted, Lawrenceville, NJ, USA

R. Ionescu-Ittu · H. Romdhani · A. Guerin Analysis Group, Montreal, QC, Canada

P. Kessler · M. Borentain · K. Friend · M. DeSouza Bristol Myers Squibb, Lawrenceville, NJ, USA

N. Sato $(\square)$

Department of Cardiovascular Medicine, Kawaguchi Cardiovascular and Respiratory Hospital, Saitama, Japan

e-mail: n.sato@kheartlung.jp
Results: Of 30,360 patients (mean age $=80.0$ years; $52.2 \%$ male), $87.0 \%$ were treated during the hospitalization with IV diuretics, $63.9 \%$ with IV vasodilators, and $13.8 \%$ with intensified therapies. On average, the duration of IV therapy was 10.6 days. Inhospital cardiac rehabilitation was utilized by $51.7 \%$ of the patients for 11.7 days on average. Mean LOS was 23.3 days, while in-hospital mortality and 30-day HF readmission post-discharge were 13.2 and $9.5 \%$, respectively. Hospitalization outcomes remained stable between 2013 and 2017 despite important changes in AHF management such as a decrease in carperitide use $(55.9-40.0 \%$ in 2017), and increases in use of tolvaptan (from $14.2 \%$ in 2013 to $31.3 \%$ in 2017) and of cardiac rehabilitation (from $43.2 \%$ in 2013 to $56.1 \%$ in 2017). Patients with intensified therapies had the longest IV therapy duration (mean 23.8 days vs. 5.5-9.9 days), the highest cardiac rehabilitation services use (60.2 vs. $38.3-57.0 \%)$, the longest LOS (mean 36.7 vs. 16.3-22.2 days), and the highest in-hospital mortality (37.4 vs. $3.1-12.4 \%)$ compared to the other treatment groups.

Conclusions: Contemporary treatment for AHF hospitalization in Japan comprises a long duration of IV therapy followed by extended use of oral medications and in-hospital cardiac rehabilitation prior to discharge. Patients requiring intensified therapies had much longer LOS and higher in-hospital mortality. 
Keywords: Acute heart failure; Cardiac rehabilitation; Disease management; Hospitalization outcomes

\section{Key Summary Points}

\section{Why carry out this study?}

Prior real-world acute heart failure (AHF) studies in Japan are mostly registry studies focusing on the overall population of patients hospitalized for AHF in cardiovascular hospitals, without differentiation based on the severity of presentation, and offering limited information on AHF management during hospitalization.

This study used de-identified data from the Medical Data Vision database (2013-2017; which covers both general and cardiovascular hospitals) to provide an up-to-date real-world characterization of treatment patterns and hospitalization outcomes of patients hospitalized for AHF in Japan who had no evidence of severe presentation at admission (such as cardiogenic shock at admission or history of end-stage renal disease) and received treatment with intravenous (IV) diuretics and/or IV vasodilators as initial therapy.

\section{What was learned from the study?}

The study revealed that in patients hospitalized for AHF in Japan $(N=30,360$; mean age 80.0 years), a comprehensive and integrated treatment modality was provided during hospitalization including an average of 10.6 days from the initiation to the completion of IV therapies, an average of 13.5 days on oral diuretics/vasodilators following IV therapy in over $90 \%$ of patients, and the use of cardiac rehabilitation in $51.7 \%$ of patients for an average of 11.7 days.
A higher in-hospital mortality rate was observed $(13.2 \%)$ compared to the rates previously reported in the literature (4.7-8.7\%); patients receiving intensified therapies had the longest duration of IV therapy and highest mortality.

The study confirmed previous reports of important changes in AHF management over time in Japan, including a sharp decrease in carperitide use and increases in tolvaptan and cardiac rehabilitation use from 2013 to 2017.

In Japan, the contemporary treatment approach in patients hospitalized for AHF comprises a long duration of IV therapy followed by extended use of oral medications and in-hospital cardiac rehabilitation prior to discharge. Differences in treatments were related to different outcomes. These results supplement the existing registries.

\section{DIGITAL FEATURES}

This article is published with digital features, a summary slide, to facilitate understanding of the article. To view digital features for this article go to https://doi.org/10.6084/m9. figshare.13583027.

\section{INTRODUCTION}

Patients hospitalized for acute heart failure (AHF), characterized by a rapid onset of new or worsening HF signs and symptoms, have a poor prognosis and a high risk of readmission and death post-discharge [1]. The main therapeutic options currently available to these patients include intravenous (IV) diuretics, vasodilators, vasoconstrictors, and inotropic agents [2, 3]. However, the management of patients hospitalized for AHF remains challenging given the highly heterogeneous patient populations and the limitations of the currently available therapies $[4,5]$. Indeed, while the available therapies 
can relieve symptoms, they have limited longterm survival benefits and may be associated with short- and long-term adverse effects, which include impairment of renal function and hypotension $[2,6]$.

Several previous studies have shown that the in-hospital AHF management in Japan is different from that of other countries [2, 7-9]. Notably, patients stay in hospitals much longer in Japan than in the United States (US) and Europe (e.g., median range: 14-21 days in Japan $[10,11]$ vs. $4-5$ days in the US [12] and 8-10 days across Europe [9]). Furthermore, trends in natriuretic peptides and vasopressin receptor antagonists' usage vary considerably between Japan and the US. Specifically, tolvaptan is approved and broadly used for the treatment of AHF in Japan, but it is only approved for the treatment of hyponatraemia in the US [2].

A number of recent Japanese studies, such as registry-based studies [10], electronic medical records [EMR]/claims studies [13-17], and retrospective chart review studies [18], have investigated treatment patterns and/or outcomes during AHF hospitalizations in Japan. Nevertheless, gaps in knowledge remain. Most of the previous research studied overall populations of patients hospitalized for AHF without differentiation based on severity of presentation. However, treatment strategies and associated outcomes of patients who are hemodynamically stable at admission and those who are not could be very different. Moreover, previous studies offer limited details regarding how patients were managed during long hospital stays, such as the type and duration of IV therapies, and treatment of patients after IV therapy and before discharge. In particular, there is very limited information regarding the utilization of in-hospital cardiac rehabilitation in Japan. Cardiac rehabilitation is a Class IIa recommendation for AHF patients once their condition stabilizes based on evidence that it decreases all-cause mortality, according to the Japanese Circulation Society (JCS) Guidelines for Rehabilitation in Patients with Cardiovascular Disease [19]. Therefore, the objective of this study was to gain a granular understanding of patient characteristics, disease management, and hospitalization outcomes of patients hospitalized for AHF in Japan who are hemodynamically stable at presentation (e.g., patients without cardiogenic shock at admission or history of end-stage renal disease [ESRD]).

\section{METHODS}

\section{Data Source}

This retrospective cohort study used data from the Japan Medical Data Vision (MDV) database that includes health claims and diagnosis procedure combination (DPC) EMR records from 376 Japanese DPC hospitals, representing 21.7\% of all the 1730 DPC hospitals in Japan [20]. The MDV database covers $\sim 20$ million patients in Japan representing $\sim 15 \%$ of Japan's population. Data recorded in the MDV database include International Classification of Diseases, 10th Revision (ICD-10) diagnosis codes, disease names coded using Japanese-specific disease codes, and procedures and drug prescriptions and administration coded using Japanesespecific receipt codes. In-hospital mortality information is available in the EMR data.

\section{Compliance with Ethics Guidelines}

Data are de-identified and comply with the patient confidentiality requirements of the US Health Insurance Portability and Accountability Act. No institutional review board approval was required for this study.

\section{Study Sample}

Adult patients hospitalized for AHF between 2013 and 2017 were selected (the five most recent complete years available, in order to provide a contemporary view of AHF disease management and outcomes in Japan; year 2018 was not included because only 2 months of data were available for this year). 'AHF hospitalizations' were defined as DPC hospitalizations of $\geq 2$ days and $<6$ months, for which a confirmed HF diagnosis (ICD-10 diagnosis codes: I50.x) was specified as "the reason behind the 
hospitalization". Patients with eligible AHF hospitalizations were required to have data available for $\geq 6$ months pre-admission and $\geq 30$ days post-discharge. To further support $\mathrm{HF}$ as the reason for the hospitalization and consistent with existing clinical trial endpoint definitions of AHF hospitalization events, patients were included only if they were treated with IV diuretics and/or IV vasodilators within $48 \mathrm{~h}$ of admission. Given the intent of this study to inform care for the large majority of patients with HF who are hemodynamically stable at presentation, patients with acute coronary syndrome during the AHF hospitalization and patients with the following criteria were excluded: (1) history of ESRD or evidence of ESRD at admission; and (2) severe acute $\mathrm{HF} /$ cardiogenic shock, proxied by either the use of an intensified therapy on the admission day (i.e., mechanical support [including respiratory support using artificial devices with intubation, circulatory support including percutaneous cardiopulmonary support/extracorporeal membrane oxygenation, intra-aortic balloon pumping, and artificial devices, and renal replacement therapy], IV inotropic agents, or vasoconstrictors), or by evidence of low systolic blood pressure (SBP) at admission (SBP $<100 \mathrm{mmHg}$ was used, based on an inherent cut-off in the data: SBP $<$ or $\geq 100 \mathrm{mmHg}$ ) (Supplemental Table 1). If during the 5-year study period a patient had multiple AHF hospitalizations that satisfied the study inclusion/exclusion criteria, one eligible AHF hospitalization was randomly selected as the index AHF admission, and the index date was defined as the admission date of the index hospitalization. By randomly choosing the hospitalization, over-representation of de novo or pre-existing HF would be avoided and the selected hospitalizations would be more representative of the current practice for AHF hospitalizations in Japan.

\section{Measurements}

Patient characteristics were defined on or prior to the index date, as summarized below: demographics at the index date, Charlson
Comorbidity Index (CCI) [21] and the individual comorbidities assessed in the year pre-index, whether or not patients were de novo HF on admission to the index hospitalization (defined as the absence of HF diagnoses any time preindex), and whether or not patients had an AHF hospitalization in the year before the index date (defined as hospitalizations with HF diagnosis and therapies for the treatment of AHF [see Supplemental Table 2] received within $48 \mathrm{~h}$ of admission; the definition relies on evidence that AHF therapies are usually initiated during the first 2 days of hospitalization $[11,22]$ and on AHF guidelines that emphasize the importance of immediate diagnosis and treatment of patients presenting with AHF [3]).

Use of medications during the index AHF hospitalization included: (1) duration of IV therapy, measured as the time from the first to the last IV therapy of any IV therapies for AHF (i.e., diuretics, vasodilators, vasoconstrictors, and inotropic agents; Supplemental Table 2), as a proxy for the time needed to hemodynamically stabilize the patient; (2) use of IV diuretics, such as days on IV diuretics, dose increase (defined as an increase in number of vials) and combination of an IV diuretic with another type of IV/oral diuretic (i.e., loop diuretics, thiazide, potassium-sparing [including spironolactone], or the aquaretic tolvaptan) on the same day; (3) use of IV vasodilators, such as days of IV vasodilator treatment and dose increase (defined as an increase in number of vials); (4) use of oral diuretics or oral vasodilators after IV therapy and before discharge (this was conducted in patients who were discharged alive); (5) use of mechanical support -mechanical ventilation with intubation, mechanical circulation, renal replacement therapy- during the index hospitalization (Supplemental Table 2); and (6) use of and duration of cardiac rehabilitation services. The proportions of patients with records for oral therapies for $\mathrm{HF}$ at admission (i.e., during the first two days of hospitalization) and at discharge (i.e., on the discharge day; among patients discharged alive) were reported (including angiotensin-converting-enzyme inhibitors [ACEi], angiotensin receptor blockers [ARBs], mineralocorticoid receptor antagonists [MRAs], oral diuretics, and oral vasodilators; 
Supplemental Table 2). Hospitalization outcomes were measured during the index AHF hospitalization and up to 30 days post-discharge and included, (1) length of stay (LOS), (2) use and duration of use of intensive care unit (ICU) services, (3) in-hospital mortality, and (4) 30-day post-discharge HF-readmission (defined as hospital readmission with therapies for the treatment of AHF (see Supplemental Table 2) received within $48 \mathrm{~h}$ of admission.

Temporal trends (from 2013 to 2017) were reported for the use of selected AHF medications (used by $>10.0 \%$ of patients), use of cardiac rehabilitation, and AHF hospitalization outcomes.

In addition, patients were categorized into four mutually exclusive treatment groups based on the type of AHF therapies received during the index AHF hospitalization: (1) intensified therapies (mechanical support, inotropic agents, or vasoconstrictors on day 2 or later during the hospitalization); (2) IV diuretics only; (3) IV vasodilators only; and (4) both IV diuretics and IV vasodilators (see Supplemental Table 2). Patient characteristics, treatment patterns, and outcomes were described for each group.

\section{Statistical Analysis}

All analyses were descriptive and no statistical testing was performed. For all analyses, means, standard deviations, and medians were reported for continuous variables; frequencies and proportions were reported for categorical variables.

\section{RESULTS}

\section{Study Sample}

Of 56,390 adults with $\geq 1$ DPC hospitalization with data available for $\geq 6$ months pre-admission and $\geq 30$ days post-discharge, 30,360 patients met the selection criteria in years 2013-2017 and were included in the study sample (Supplemental Fig. 1).

\section{PATIENT BASELINE CHARACTERISTICS}

The mean age of the 30,360 patients included in the study was 80.0 years, with $40.8 \%$ of patients aged $\geq 85$ years; $52.2 \%$ were male. There were $22.5 \%$ of patients with de novo HF when admitted to the hospital. Among those with existing HF, 35.2\% had an HF-related hospitalization in the year pre-admission. Mean CCI was 3.8. Cardiovascular comorbidities were present in $83.0 \%$ of patients, and included hypertension $(69.5 \%)$, coronary artery disease (51.4\%), cardiac arrhythmias (49.1\%), dyslipidemia $(41.0 \%)$, and valvular disease (30.5\%). Other comorbidities with a prevalence $>10.0 \%$ included cancer $(31.6 \%)$, chronic peptic ulcer disease $(26.3 \%)$, fluid and electrolyte disorders $(25.7 \%)$, diabetes $(25.0 \%)$, deficiency anemia (25.0\%), and chronic obstructive pulmonary disease (24.7\%; Table 1).

\section{In-Hospital AHF Therapy and Cardiac Rehabilitation}

Overall, IV therapy with diuretics and vasodilators was used by 87.0 and $63.9 \%$ of patients, respectively (Table 2), while intensified therapies (i.e., IV vasoconstrictors, inotropic agents, or mechanical support) were used by $13.8 \%$ of patients. On average, the duration of IV therapies was 10.6 days (median of 6 days). Among those who received IV diuretics, almost all patients were started with furosemide (99.5\%), with a mean initial dose of $31.7 \mathrm{mg} /$ day and a median of $20 \mathrm{mg} /$ day; further, $27.0 \%$ experienced dose increase and $45.2 \%$ took a combination of two different types of diuretics, including $19.1 \%$ who used a combination of an IV diuretic with tolvaptan. Patients were on IV diuretics for 6.8 days on average. Among those who received IV vasodilator, the majority of patients were started with carperitide (70.0\%). Further, 20.0\% of patients experienced a dose increase. Patients were on IV vasodilators for 5.5 days on average. After discontinuation of IV therapy and prior to discharge, $90.5 \%$ of patients received diuretics 
Table 1 Baseline characteristics

\begin{tabular}{|c|c|}
\hline Demographics & $\begin{array}{l}\text { Patients with AHF } \\
\text { hospitalization } \\
N=30,360\end{array}$ \\
\hline $\begin{array}{l}\text { Age at admission (years), mean } \\
\text { [median] }\end{array}$ & $80.0[83.0]$ \\
\hline \multicolumn{2}{|l|}{ Age, $N(\%)$} \\
\hline $18-54$ years & $856(2.8 \%)$ \\
\hline $55-64$ years & $1527(5.0 \%)$ \\
\hline $65-74$ years & $4628(15.3 \%)$ \\
\hline $75-84$ years & $10,968(36.1 \%)$ \\
\hline Age 85 years or older, $N(\%)$ & $12,381(40.8 \%)$ \\
\hline Male, $N(\%)$ & $15,860(52.2 \%)$ \\
\hline $\begin{array}{l}\text { BMI at admission, mean } \\
\text { [median }]\end{array}$ & $22.9[22.3]$ \\
\hline \multicolumn{2}{|l|}{ HF history } \\
\hline De novo $\mathrm{HF}^{\mathrm{a}}, N(\%)$ & $6826(22.5 \%)$ \\
\hline \multicolumn{2}{|l|}{$\begin{array}{l}\text { Hospitalization in the year pre- } \\
\text { admission }\end{array}$} \\
\hline All-cause, $N(\%)$ & $13,525(44.5 \%)$ \\
\hline $\begin{array}{l}\text { HF-related, } N(\% \text { with existing } \\
\mathrm{HF})^{\mathrm{b}}\end{array}$ & $8284(35.2 \%)$ \\
\hline \multicolumn{2}{|l|}{ Comorbidities $^{c}$} \\
\hline CCI, mean [median] & $3.8[3.0]$ \\
\hline $\begin{array}{l}\text { Cardiovascular comorbidities, } \mathrm{N} \\
(\%)\end{array}$ & $25,188(83.0 \%)$ \\
\hline Hypertension & $21,112(69.5 \%)$ \\
\hline Cardiac arrhythmias & $14,913(49.1 \%)$ \\
\hline Peripheral vascular disorder & $6484(21.4 \%)$ \\
\hline Valvular disease & $9262(30.5 \%)$ \\
\hline Coronary artery disease & $15,608(51.4 \%)$ \\
\hline Stroke ${ }^{\mathrm{d}}$ & $5875(19.4 \%)$ \\
\hline Pulmonary circulation disorder & $1393(4.6 \%)$ \\
\hline Dyslipidemia $^{\mathrm{e}}$ & $12,443(41.0 \%)$ \\
\hline \multicolumn{2}{|l|}{$\begin{array}{l}\text { Other comorbidities } \\
\quad(\text { prevalence }>10 \%), N(\%)\end{array}$} \\
\hline Diabetes & $7603(25.0 \%)$ \\
\hline
\end{tabular}

Table 1 continued

\begin{tabular}{ll}
\hline Demographics & $\begin{array}{l}\text { Patients with AHF } \\
\text { hospitalization } \\
\boldsymbol{N}=\mathbf{3 0 , 3 6 0}\end{array}$ \\
\hline CKD (excl. ESRD) & $6163(20.3 \%)$ \\
Cancer & $9608(31.6 \%)$ \\
Chronic peptic ulcer disease & $7977(26.3 \%)$ \\
Fluid and electrolyte disorders & $7793(25.7 \%)$ \\
Deficiency anemia & $7599(25.0 \%)$ \\
Chronic pulmonary disease ${ }^{\mathrm{g}}$ & $7534(24.8 \%)$ \\
Chronic obstructive & $7490(24.7 \%)$ \\
pulmonary disease & \\
Liver disease & $6340(20.9 \%)$ \\
Coagulopathy & $4828(15.9 \%)$ \\
Hypothyroidism & $3240(10.7 \%)$ \\
\hline
\end{tabular}

$A H F$ acute heart failure, $B M I$ body mass index, $C C I$ Charlson Comorbidity Index, $C K D$ chronic kidney disease, $E S R D$ end-stage renal disease, $H F$ heart failure

${ }^{a}$ No AHF diagnosis anytime pre-admission

b HF-related hospitalizations were defined as hospitalizations with $\geq 1 \mathrm{HF}$ drug treatment received during the first two days of the hospitalization

${ }^{c}$ Evaluated in the 12 months before the index date; only comorbidities with $\geq 10 \%$ prevalence were reported

d Included subarachnoid hemorrhage, intracerebral hemorrhage, cerebral infarction, and other stroke

e Included disorders of lipoprotein metabolism and other lipedema

${ }^{f}$ Included hypertensive chronic kidney disease, chronic kidney disease, unspecified renal failure. Patients with a diagnosis for ESRD prior to hospitalization were excluded by design

g Included chronic pulmonary heart disease (excluding primary pulmonary hypertension, pulmonary embolism, kyphoscoliotic heart disease), chronic obstructive pulmonary disease and allied conditions (e.g., asthma, bronchitis, emphysema), pneumoconiosis and other lung diseases due to external agents 
Table 2 Therapy use during the AHF hospitalization ${ }^{a}$

Patients with AHF hospitalization $N=30,360$

\section{IV therapy during the AHF} hospitalization

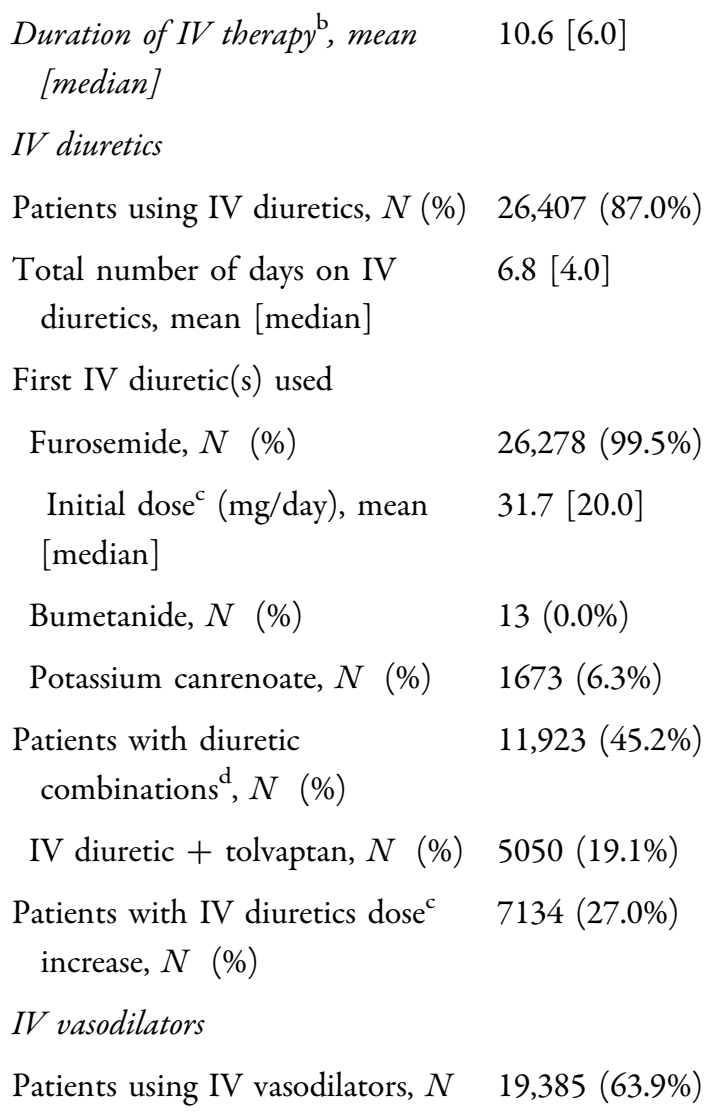
(\%)

Total number of days on IV $\quad 5.5$ [4.0] vasodilators, mean [median]

First IV vasodilator(s) used, $N$ (\%)

\begin{tabular}{ll} 
Carperitide & $13,567(70.0 \%)$ \\
Isosorbide dinitrate & $3200(16.5 \%)$ \\
Nicorandil & $580(3.0 \%)$ \\
Nitroglycerin & $4273(22.0 \%)$ \\
Nitroprusside & $20(0.1 \%)$ \\
Patients with IV vasodilators dose & $3791(19.6 \%)$ \\
increase, $N(\%)$ & \\
\hline
\end{tabular}

Table 2 continued

Patients with AHF hospitalization $N=30,360$
From last $I V$ dose to discharge,

$$
\text { among 26,365 patients }
$$$$
\text { discharged alive }
$$

Patients using oral

diuretics/vasodilators, $N$ (\%)

Number of days on oral

$13.5[10.0]$

diuretics/vasodilators, mean

[median]

Percent of days on oral diuretics/vasodilators, mean [median]

\section{Mechanical support ${ }^{\mathrm{e}}$ during the}

\section{AHF hospitalization}

Patients using mechanical support, 1447 (4.8\%)

$$
\text { N (\%) }
$$

Mechanical ventilation with intubation

Mechanical circulation

583 (1.9\%)

Renal replacement therapy

$606(2.0 \%)$

$531(1.7 \%)$

Oral HF therapies at admission

\section{and discharge}

At admission

ACE inhibitors

$3514(11.6 \%)$

ARBs

$5830(19.2 \%)$

Beta blockers

7986 (26.3\%)

MRA (i.e., eplerenone) ${ }^{\mathrm{f}}$

$695(2.3 \%)$

Oral diuretics ${ }^{\mathrm{e}}$

$13,916(45.8 \%)$

Loop

$10,579(34.8 \%)$

Thiazide

883 (2.9\%)

Potassium-sparing ${ }^{\mathrm{f}}$

5493 (18.1\%)

Vasopressin receptor antagonist

3842 (12.7\%)

Oral vasodilators ${ }^{\mathrm{e}}$
$1654.0(5.4 \%)$ 
Table 2 continued

\section{Patients with AHF hospitalization $N=30,360$}

\section{At discharge, among 26,365 \\ patients discharged alive}

\begin{tabular}{|c|c|}
\hline ACE inhibitors & $5398(20.5 \%)$ \\
\hline ARBs & $8167(31.0 \%)$ \\
\hline Beta blockers & $13,066(49.6 \%)$ \\
\hline MRA (i.e., eplerenone) ${ }^{\mathrm{f}}$ & $1092(4.1 \%)$ \\
\hline Oral diuretics ${ }^{\mathrm{e}}$ & $22,009(83.5 \%)$ \\
\hline Loop & $20,633(78.3 \%)$ \\
\hline Thiazide & $1590(6.0 \%)$ \\
\hline Potassium-sparing $^{f}$ & $9024(34.2 \%)$ \\
\hline Vasopressin receptor antagonist & $3398(12.9 \%)$ \\
\hline Oral vasodilators ${ }^{\mathrm{e}}$ & $2252(8.5 \%)$ \\
\hline $\begin{array}{l}\text { Cardiac rehabilitation during the } \\
\text { AHF hospitalization }\end{array}$ & \\
\hline $\begin{array}{l}\text { Patients using cardiac } \\
\text { rehabilitation services, } N \text { (\%) }\end{array}$ & $15,705(51.7 \%)$ \\
\hline $\begin{array}{l}\text { Number of days with cardiac } \\
\text { rehabilitation services (excl. } \\
\text { gaps), mean [median] (among } \\
\text { patients with cardiac } \\
\text { rehabilitation) }\end{array}$ & $11.7[9.0]$ \\
\hline
\end{tabular}

$A C E$ angiotensin-converting enzyme, $A H F$ acute heart failure, $A R B$ angiotensin receptor blockers, $H F$ heart failure, $I V$ intravenous, $I C U$ intensive care unit, $M R A$ mineralocorticoid receptor antagonists, $S D$ standard deviation ${ }^{a}$ See the list of therapies in Supplemental Table 2

${ }^{b}$ Includes gaps in use of IV therapy

${ }^{c}$ IV dosages were calculated based on the number of used vials reported in the data

d Administration in the same day of two different types of diuretics, of which $\geq 1$ was IV

e Mechanical support includes mechanical ventilation with intubation, mechanical circulation, and renal replacement therapy

${ }^{\mathrm{f}}$ Based on the mechanism of action, spironolactone was classified in this study as a potassium-sparing diuretic and not as an MRA and/or vasodilators in oral formulation for an average of 13.5 days.

At admission, oral diuretics were the most commonly used oral medication (45.8\%, including $34.8 \%$ loop diuretics), followed by beta blockers (26.3\%) and ARBs (19.2\%). Oral diuretics were also the most commonly used oral medications at discharge $(83.5 \%$, including $78.3 \%$ loop diuretics), followed by beta blockers (49.6\%) and ARBs (31.0\%; Table 2).

During hospitalization, cardiac rehabilitation services were used by $51.7 \%$ of patients. Among them, 58.0\% started cardiac rehabilitation before the end of IV therapy. The average number of days with cardiac rehabilitation was 11.7 days either consecutively or in intermittent fashion, and patients could have more than one record of cardiac rehabilitation services on one day (Table 2). Patients who used cardiac rehabilitation services were older ( 81.3 vs. 78.6 years) and had lower CCI (3.7 vs. 3.9) than those who did not use cardiac rehabilitation services. Compared to patients who did not use cardiac rehabilitation services during hospitalization, a higher proportion of patients who used cardiac rehabilitation services received intensified therapies (16.0 vs. $11.3 \%)$ and had an ICU stay (25.7 vs. $15.8 \%$ ); furthermore, patients who used cardiac rehabilitation services had a longer duration of IV therapy (mean 12.5 vs. 8.5 days) and a longer stay in hospital (mean 28.5 vs. 17.8 days). Nevertheless, patients who used cardiac rehabilitation services had a lower in-hospital mortality rate compared to those who did not use these services (10.9 vs. 15.6\%) (Table 3).

\section{Hospitalization Outcomes}

The average LOS was 23.3 days, with $40.1 \%$ of patients staying in hospital between 15 and 30 days, and $21.5 \%$ hospitalized for $\geq 31$ days (median LOS was 17 days). About one in five patients $(20.9 \%)$ were in the ICU for an average of 5.3 days. The rate of in-hospital mortality was $13.2 \%$. Among patients who were discharged alive, $9.5 \%$ of patients were readmitted for AHF within 30-days post-discharge (Fig. 1). 
Table 3 Cardiac rehabilitation

\begin{tabular}{|c|c|c|c|c|}
\hline & \multicolumn{4}{|l|}{ All patients } \\
\hline & \multicolumn{4}{|l|}{$N=30,360$} \\
\hline & \multicolumn{2}{|c|}{$\begin{array}{l}\text { With cardiac rehabilitation } \\
\text { during the index } \\
\text { hospitalization }\end{array}$} & \multicolumn{2}{|c|}{$\begin{array}{l}\text { Without cardiac } \\
\text { rehabilitation during the } \\
\text { index hospitalization }\end{array}$} \\
\hline & $N=15,705$ & $(51.7 \%)$ & $N=14,655$ & $(48.3 \%)$ \\
\hline \multicolumn{5}{|l|}{ Patients characteristics, $N(\%)$} \\
\hline Age (years), mean [median] & 81.3 & {$[84.0]$} & 78.6 & {$[81.0]$} \\
\hline Age 85 years or older, $N(\%)$ & 7195 & $(45.8 \%)$ & 5186 & $(35.4 \%)$ \\
\hline Male, $N(\%)$ & 7733 & $(49.2 \%)$ & 8127 & $(55.5 \%)$ \\
\hline De novo $\mathrm{HF}, N$ (\%) & 3744 & $(23.8 \%)$ & 3082 & $(21.0 \%)$ \\
\hline Charlson Comorbidity Index, mean $\pm \mathrm{SD}$ [median] & 3.7 & $\pm 2.5[3.0]$ & 3.9 & $\pm 2.6[4.0]$ \\
\hline Cardiovascular comorbidities & 12,810 & $(81.6 \%)$ & 12,378 & $(84.5 \%)$ \\
\hline Hypertension & 10,708 & $(68.2 \%)$ & 10,404 & $(71.0 \%)$ \\
\hline Cardiac arrhythmias & 7576 & $(48.2 \%)$ & 7337 & $(50.1 \%)$ \\
\hline Peripheral vascular disorder & 3412 & $(21.7 \%)$ & 3072 & $(21.0 \%)$ \\
\hline Valvular disease & 4652 & $(29.6 \%)$ & 4610 & $(31.5 \%)$ \\
\hline Coronary artery disease & 7900 & $(50.3 \%)$ & 7708 & $(52.6 \%)$ \\
\hline Stroke & 3055 & $(19.5 \%)$ & 2820 & $(19.2 \%)$ \\
\hline Pulmonary circulation disorder & 660 & $(4.2 \%)$ & 733 & $(5.0 \%)$ \\
\hline Dyslipidemia & 6297 & $(40.1 \%)$ & 6146 & $(41.9 \%)$ \\
\hline \multicolumn{5}{|l|}{ Treatments during the index hospitalization } \\
\hline Use of intensified therapies, $N(\%)$ & 2515 & $(16.0 \%)$ & 1662 & $(11.3 \%)$ \\
\hline Duration of IV therapy, mean [median] & 12.5 & {$[7.0]$} & 8.5 & {$[5.0]$} \\
\hline \multicolumn{5}{|l|}{ Outcomes } \\
\hline Length of stay, mean $\pm \mathrm{SD}$ [median] & 28.5 & $\pm 22.2[21.0]$ & 17.8 & $\pm 16.3[14.0]$ \\
\hline ICU stay during the index hospitalization, $N(\%)$ & 4034 & $(25.7 \%)$ & 2320 & $(15.8 \%)$ \\
\hline In-hospital mortality, $N$ (\%) & 1707 & $(10.9 \%)$ & 2289 & $(15.6 \%)$ \\
\hline $\begin{array}{l}\text { HF-rehospitalization } 30 \text { days post-discharge, } N \quad \text { (\% } \\
\text { discharged alive) }\end{array}$ & 1380 & $(9.9 \%)$ & 1123 & $(9.1 \%)$ \\
\hline
\end{tabular}

$I V$ intravenous, $H F$ heart failure, $I C U$ intensive care, $S D$ standard deviation 

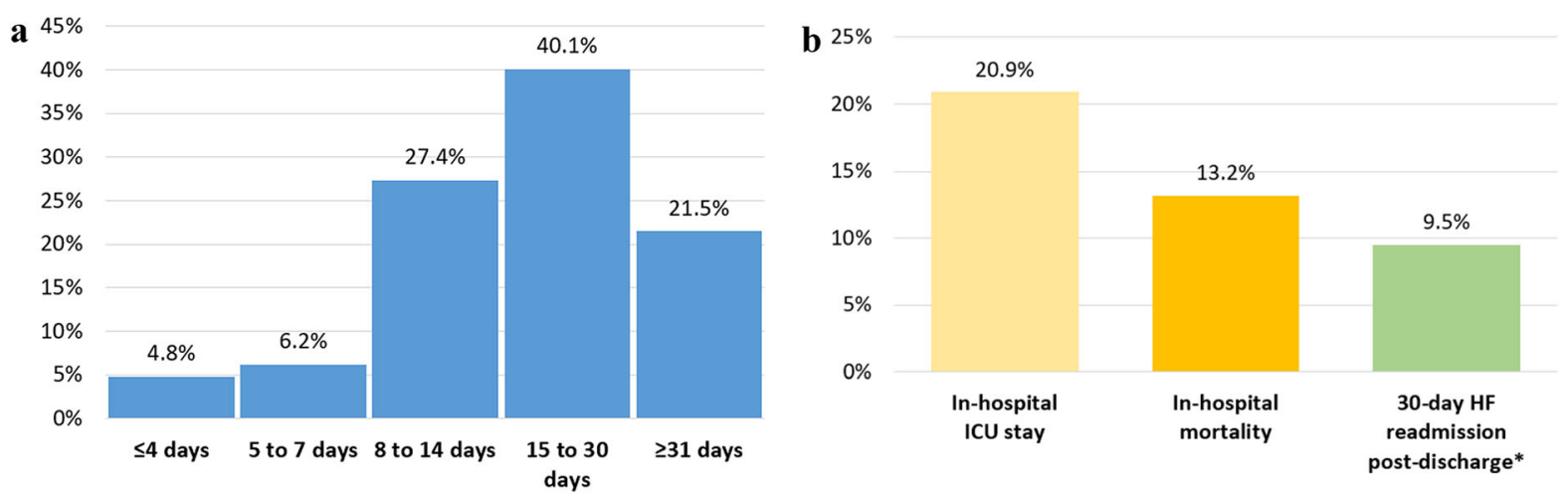

Fig. 1 a Length of stay for the index hospitalization. $\mathbf{b}$ In-hospital and post-discharge outcomes. $H F$ heart failure, ICU intensive care unit. Asterisk indicates estimated among patients discharged alive

Temporal Trends in Use of AHF

Medication, Use of Cardiac Rehabilitation, and Hospitalization Outcomes

Temporal trends in the use of AHF agents during hospitalizations showed a decrease in IV carperitide use from $55.9 \%$ in 2013 to $40.0 \%$ in 2017 , in contrast with an increase in oral tolvaptan use from $14.2 \%$ in 2013 to $31.3 \%$ in 2017. During the same time period, the use of IV furosemide, IV isosorbide dinitrate (data not shown), and IV nitroglycerin (data not shown) remained relatively stable. Over the same period, LOS decreased from 24.6 to 21.9 days and AHF in-hospital mortality decreased from 15.7 to $11.9 \%$. Thirty-day HF readmission post-discharge decreased slightly from 9.5 to $8.4 \%$ from 2013 to 2014 but rose to $10.9 \%$ by 2017 . The proportion of patients using cardiac rehabilitation services rose from $43.2 \%$ in 2013 to $56.1 \%$ in 2017 (Fig. 2).

\section{Patient Characteristics, Treatments, and Outcomes by Treatment Group}

Across the four treatment groups, mean age at admission ranged from 77.3 years (the IV vasodilators only group; IV-Vaso) to 81.6 years (the IV diuretics only group; IV-Diur); the proportion of patients $\geq 85$ years ranged from $28.8 \%$ (IV-Vaso) to $48.9 \%$ (IV-Diur); while the proportion of de novo HF ranged from 15.6\% (IV-Vaso) to $25.2 \%$ (both IV vasodilators and IV diuretics groups; IV-Diur-Vaso).
Compared to the three groups of patients who did not receive intensified therapies, patients who received intensified therapies (INTENS) had the longest duration of IV therapy (mean 23.8 vs. 5.5-9.9 days) and were the most likely to experience a dose increase for both IV diuretics (51.4 vs. $21.6-23.9 \%$ ) and IV vasodilators (27.6 vs.13.1-19.4\%); similarly, patients in the INTENS group had the highest use of cardiac rehabilitation services during hospitalization (60.2 vs. $38.3-57.0 \%)$, longest LOS (mean 36.7 vs. 16.3-22.2 days), highest utilization of ICU services $(39.2$ vs. 11.8-24.1\%), and the highest rate of in-hospital mortality (37.4 vs. $3.1-12.4 \%$; Table 4 ).

\section{DISCUSSION}

This study described details on how patients hospitalized for AHF were managed during hospitalization, and the outcomes associated with different treatments in Japan. Since patients who are not hemodynamically stable at admission could be treated very differently and may have distinctive outcomes, this study focused on those who are hemodynamically stable at admission. The study shows that in this old population (mean 80.0 years), a comprehensive and integrated treatment modality was provided during hospitalization, including an average of 10.6 days from the initiation to the completion of IV therapies, an average of 13.5 days on oral diuretics/vasodilators following IV therapy in over $90 \%$ of patients, and the 


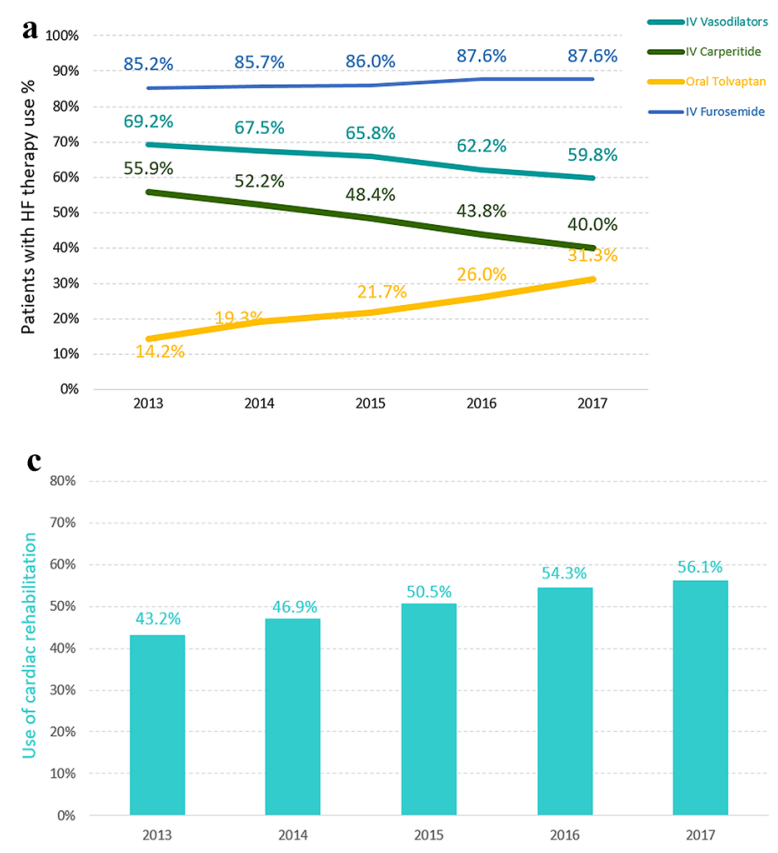

Fig. 2 Temporal trends for therapies and outcomes. a Temporal trends in use of selected HF therapies during AHF hospitalizations (agents used by $>10 \%$ of patients). b Temporal trends in LOS and AHF hospitalization outcomes. c Temporal trends in cardiac rehabilitation services during AHF hospitalizations. d Temporal trends

use of cardiac rehabilitation in $51.7 \%$ of patients for an average of 11.7 days. The mean LOS was 23.3 days, in-hospital mortality was $13.2 \%$, and 30-day HF readmission post-discharge was $9.5 \%$. Notably, patients receiving intensified therapies had the longest duration of IV therapy and highest mortality. Temporal trends show that hospitalization outcomes remained relatively stable between 2013 and 2017, despite important changes in AHF management, such as a sharp decrease in carperitide use and increases in tolvaptan and cardiac rehabilitation use.

To our knowledge, this is the first real-world study detailing treatments and outcomes in patients hospitalized for AHF in Japan. The strength of the research is several folds. First, this study used data collected from Japan DPC hospitals, which included both hospitals specialized and not specialized in treating cardiovascular diseases. Therefore, findings of this study likely reflect the overall practice of
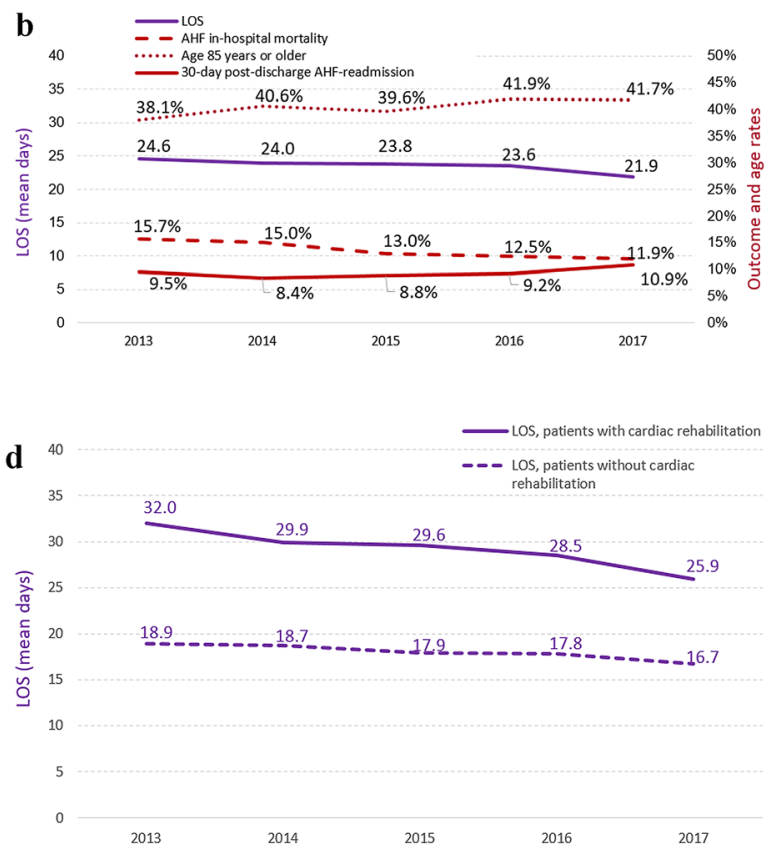

in LOS in patients with and without cardiac rehabilitation services during AHF hospitalizations. $A H F$ acute heart failure, $H F$ heart failure, $I V$ intravenous, $L O S$ length of stay

treating AHF patients in Japan, as a supplement to the findings of the existing Japan registries which predominantly included specialized cardiovascular hospitals. Nonetheless, the use of AHF therapies observed in this study is largely consistent with the registry study by Yaku et al., which reported treatment with IV furosemide in $84 \%$ of patients and IV vasodilators in $68 \%$ of patients [10]. This same study also reported similar results for the oral diuretics, ACE inhibitors, and ARBs at discharge [10]. For beta blockers, however, the discharge rates reported by Yaku et al. are higher (66 vs. $43.0 \%$ in the current study), which may be due to the inclusion of different types of hospitals in centerbased registry versus EMR/claims studies.

Second, this study reported the duration of in-hospital IV therapy, which, to our knowledge, has not been previously described. If the duration of in-hospital IV therapy can be considered as a proxy for the time needed to hemodynamically stabilize patients, it could 


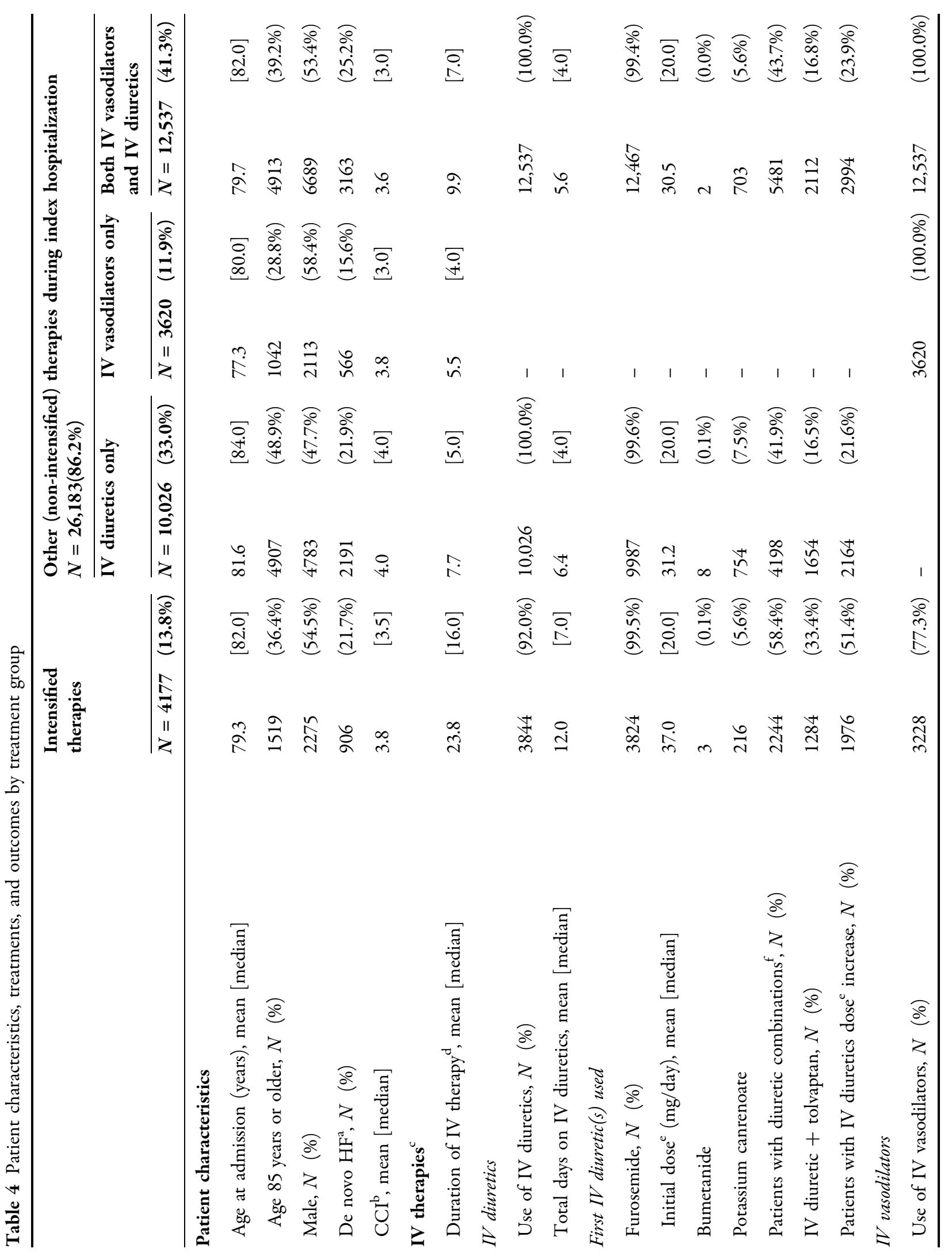




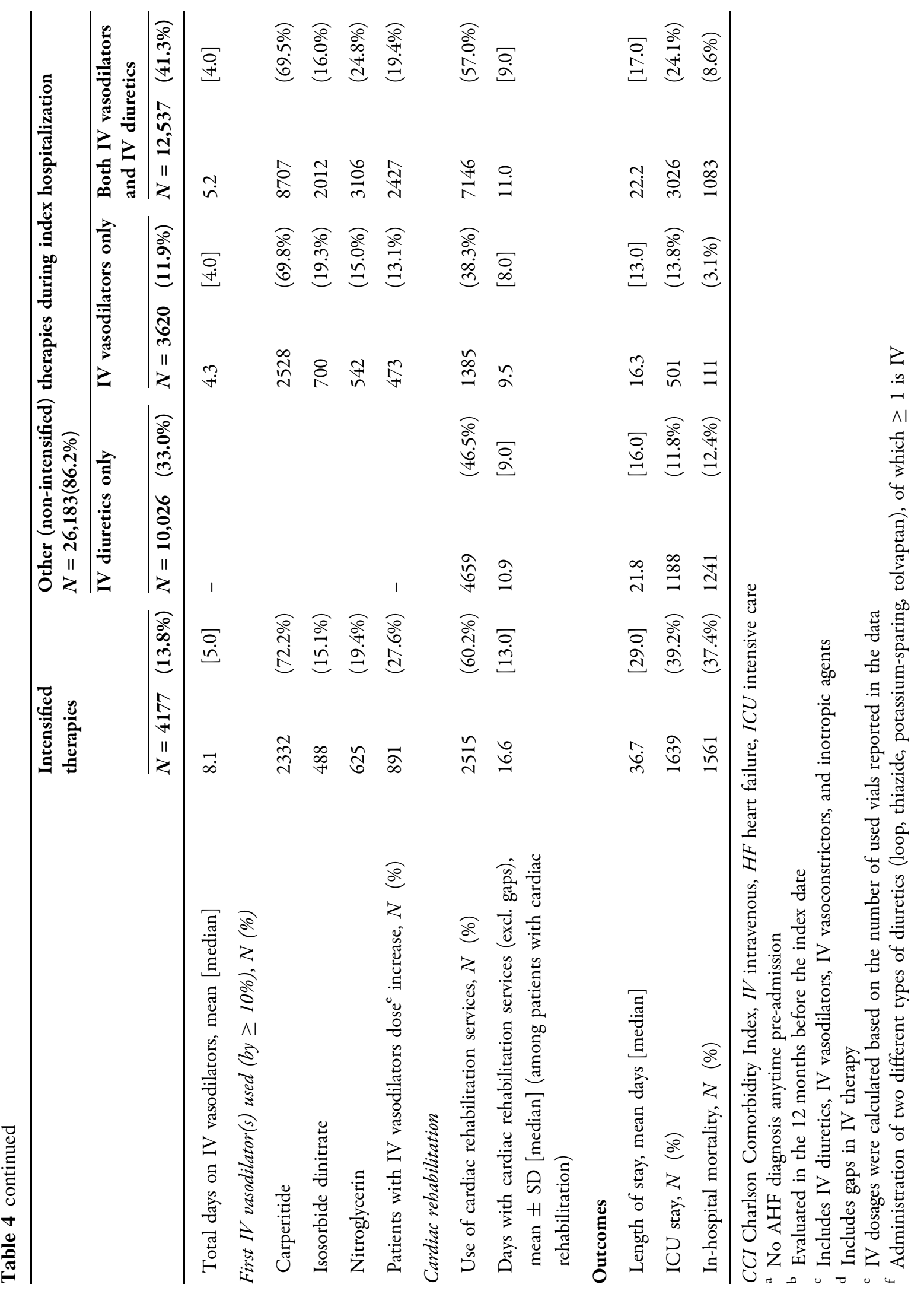


serve as a useful measure for future studies. Third, this study provided details on cardiac rehabilitation services use during hospitalization in AHF patients. Although cardiac rehabilitation is a Class IIa recommendation in the JCS guideline, information about its usage has been limited. This study showed that only $51.7 \%$ of patients used cardiac rehabilitation services during hospitalization, and that these patients were older with a more complicated clinical course during hospitalization (e.g., had longer duration of IV therapy, a higher proportion of patients received intensified therapy and had ICU stay), but had lower baseline CCI and lower in-hospital mortality. Finally, the present study covered a more recent study period and thus better reflects the current disease management and outcomes of patients hospitalized in Japan.

Consistent with previous Japanese research $[10,11,13,18,23-25]$, the present study shows that LOS is long for AHF hospitalizations in Japan (median of 17 days; mean 23 days) compared to Europe where registry-based studies showed a median AHF hospitalization LOS of 7 to 10 days [7] and to the US where a recent realworld study reported a median AHF hospitalization LOS of 4 days (mean of 6.6 days) [26]. This reveals that the duration of IV therapy in patients hospitalized for AHF in Japan (median 6 days; mean 10.6 days) is as long as or even longer than the duration of the full AHF hospitalization in Europe or the US. Our data suggest that a comprehensive and integrated treatment modality is provided to this older population of patients hospitalized for AHF in Japan, contributing to long LOS. Long duration of IV therapy followed by low-intensity management with oral medications and cardiac rehabilitation in treating AHF reflects a unique practice in Japan. Indeed, the Japanese health insurance systems support rehabilitation during the hospitalization, including for acute care hospitals [27]. For cardiovascular disease specifically, cardiac rehabilitation has been traditionally performed in the inpatient setting in Japan [28], and for the elderly in particular, an existing inpatient rehabilitation program aims for patients to regain sufficient independent walking capacity for ambulatory discharge. In addition, based on the JCS guidelines, it is recommended that patients hospitalized for HF are educated and prepared for their post-discharge living environment during the hospitalization. In DPC hospitals, this practice is supported by the unique lump-sum per-diembased payment system. With this payment scheme, a fixed amount of payment is allotted per patient per day to hospitals for selected services; the per-day payment amount varies depending on the patients' LOS and decreases as the stay gets longer.

Notably, despite this study's focus on patients who are hemodynamically stable at admission, a higher in-hospital mortality rate was observed $(13.2 \%)$ compared to the rates previously reported (4.7-8.7\%) $[10,11,13,18,24]$. There are several possible explanations for this. First, despite that patients in this study had similar ages as those reported in other registry-based and EMR-based Japanese studies ( $\sim 80$ years) $[10,13,18,23,24]$, patients in the current study appeared to have more comorbidities. For instance, $59.8 \%$ of patients in the current study had a CCI $\geq 3$, while other studies reported $8.6-41.0 \%$ [13, 23]. Some comorbidities were more prevalent in the current study compared to others, including malignancy $(31.6 \%$ in the current study vs. $8.0 \%$ [13] in the literature), history of stroke (19.4 vs. $16-17 \%$ [10, 29]), liver disease (20.9 vs. $2.1 \%$ [13]), and chronic pulmonary disease ( 24.8 vs. $5-10 \%[13,18,29])$; although diabetes is less prevalent in the current study compared to others $(25.0$ vs. $29-37 \%[10,13,18,29])$. Second, a high mortality rate could be due to non-cardiovascular conditions. While elucidation of the cause of death of these patients would be helpful to confirm this hypothesis, the cause of death was not available in the MDV database. Third, the high mortality rate may be due to the inclusion of hospitals not specialized in treating cardiovascular diseases. Evidence has shown that in-hospital mortality rates are lower in hospitals with a large number of subspecialty departments [30], in hospitals with more cardiologists per cardiovascular bed [16], and in areas with higher population density [17]. Furthermore, while there are no studies to date that have investigated differences in the implementation of the AHF guidelines between hospitals 
in Japan, evidence from other cardiology areas and countries indicates that such differences are likely [31, 32]. Fourth, differences in mortality may potentially be due to differences in the medical management of AHF. For example, prior real-world retrospective studies suggested that in-hospital mortality is higher among patients treated with carperitide compared with patients not treated with carperitide [13, 33], which is inconsistent with randomized trial findings of improved outcome among patients with AHF treated with carperitide [34]. Although the present study did not assess the impact of use of carperitide on in-hospital mortality rates, the proportion of patients who used carperitide during the AHF hospitalization in this study (highest: $55.9 \%$ in 2013; lowest: $40.0 \%$ in 2017) was higher than that reported in registry studies $(30-37 \%)$ [10, 13, 18]. Further studies are needed to clarify the impact of carperitide and other AHF treatments on inpatient mortality while controlling for confounding and other possible sources of bias. Finally, this study observed that the mortality rate was particularly high among patients requiring intensified therapies $(37.4 \%)$. The markedly high mortality rate and longer duration of IV therapy to achieve stabilization in AHF patients requiring intensified therapies suggest an important unmet need for these patients.

Temporal trends in treatment and outcomes of patients hospitalized for AHF reported in the current study suggest that changes in management and treatment practices in Japan have not translated into significant changes in patient outcomes. The decline in carperitide use concomitant with an increase in tolvaptan usage observed in the current study is consistent with the findings of a recent report of 9-year AHF management trends in Japan [11], which reported similar trends from 2007 to 2015. While carperitide is a recommended treatment for AHF in Japan (Class IIa recommendation) [3], the decline in its usage in recent years may be due to a growing body of real-world evidence suggesting that carperitide is associated with suboptimal clinical and economic outcomes $[11,13,15,33]$. Similarly, the increase in tolvaptan use may be due to the 2013 JCS and
Japan Heart Failure Society recommendation for the use of this drug in patients with HF and volume overload who have inadequate response to other diuretics $[2,35]$ as well as emerging literature indicating that early initiation of tolvaptan versus conventional therapy is associated with significantly lower all-cause death in patients with AHF [36]. In addition, the current study also reports a relative increase of $30 \%$ in the use of cardiac rehabilitation services (from $43 \%$ in 2013 to $56 \%$ in 2017), a trend that has not been previously assessed in the literature. Despite these important changes over time in the AHF management and treatment, neither our study, nor previous studies found trends of similar magnitude for AHF outcomes, including LOS, in-hospital mortality, and 30-day postdischarge HF readmission. Indeed, the outcome time trends observed in the current study are consistent with the results of the report of 9-year AHF management trends in Japan that showed a slight decrease in LOS between 2007 and 2015 that was not accompanied by changes in in-hospital mortality and 30-day HF readmission rates [11].

\section{Limitations}

This study is subject to some limitations. First, MDV is not a closed system, therefore, while information is expected to be complete for the index AHF hospitalization, services received outside the MDV hospitals (e.g., some hospitalization post-discharge and outpatient visits) may not have been captured, which may have led to underestimating the readmission rate. However, given the study's short follow-up (30days post-discharge), this underestimation is expected to be minimal. Second, only in-hospital deaths are captured in MDV, while postdischarge mortality outside the hospital is not collected and therefore cannot be evaluated. Third, the cause of mortality was unavailable in the database. Consequently, it remains unknown whether the high mortality observed was due to HF-related factors or comorbidities. Fourth, clinical assessment of severity was unavailable, therefore severe HF presentation at admission (i.e., patients not hemodynamically 
stable) was defined primarily based on treatments received on admission day for the index AHF hospitalization rather than clinical measurements of severity. Fifth, IV dosages were calculated based on the number of used vials reported, so dose increases were only captured if they required $\geq 1$ additional vial; this likely resulted in underestimating the proportion of patients with a dose increase. Furthermore, we cannot distinguish in the data between a planned titration and an unplanned dose increase. Sixth, laboratory test results were available for only $\sim 10 \%$ of the patients and could not be studied. In addition, left ventricular ejection fraction (LVEF) data were unavailable. While patients with reduced versus preserved EF could not be identified and described individually, the current study provides novel information on the overall status and trends for HF management in Japan. Finally, while MDV covers $>15 \%$ of Japan's population, the results may not be generalizable to the entire Japanese HF patient population.

\section{CONCLUSIONS}

In Japan, the contemporary treatment approach in patients hospitalized for AHF comprises a long duration of IV therapy followed by extended use of oral medications and in-hospital cardiac rehabilitation prior to discharge. Differences in treatments were related to different outcomes. In particular, patients requiring intensified therapies had much longer stays and higher in-hospital mortality. These results supplement the existing registries. Future research is needed to confirm the treatment patterns and outcomes observed in this study and to better understand the causes of the high in-hospital mortality among patients hospitalized for AHF in Japan.

\section{ACKNOWLEDGEMENTS}

Funding. The study and the Journal's Rapid Service Fee were supported by Bristol Myers Squibb.
Authorship. All named authors meet the International Committee of Medical Journal Editors (ICMJE) criteria for authorship for this article, take responsibility for the integrity of the work as a whole, and have given their approval for this version to be published.

Medical Writing and Editorial Assistance. Medical writing assistance was provided by Gloria DeWalt, Ph.D., Loraine Georgy, Ph.D., Christine Tam, MSc, and Sara Kaffashian, Ph.D., employees of Analysis Group, which has received consultancy fees from Bristol Myers Squibb. Arthur Voegel, M. Phil., and Yawen Liang, Ph.D., also employees of Analysis Group, contributed to the data analyses.

Disclosures. Raluca Ionescu-Ittu, Hela Romdhani, and Annie Guerin are employees of Analysis Group, which has received consultancy fees from Bristol Myers Squibb. Lei Chen is currently an employee of ADC Therapeutics SA and was an employee of Bristol Myers Squibb at the time when the research was conducted. Paul Kessler, Maria Borentain, Keith Friend, and Mary DeSouza are employees of Bristol Myers Squibb. Naoki Sato has received honoraria from Otsuka, Takeda, Ono, Bayer, Teijin, Boehringer Ingelheim, and Daiichi-Sankyo, research support from Roche Diagnostics Japan, and has served as consultant for Terumo, Otsuka, Novartis, and Bristol Myers Squibb.

Compliance with Ethics Guidelines. Data are de-identified and comply with the patient confidentiality requirements of the US Health Insurance Portability and Accountability Act. No institutional review board approval was required for this study.

Data Availability. The datasets analyzed during the current study are not publicly available due to licensing agreement with Medical Data Vision Co., Ltd.

Open Access. This article is licensed under a Creative Commons Attribution-NonCommercial 4.0 International License, which permits any non-commercial use, sharing, adaptation, distribution and reproduction in any medium 
or format, as long as you give appropriate credit to the original author(s) and the source, provide a link to the Creative Commons licence, and indicate if changes were made. The images or other third party material in this article are included in the article's Creative Commons licence, unless indicated otherwise in a credit line to the material. If material is not included in the article's Creative Commons licence and your intended use is not permitted by statutory regulation or exceeds the permitted use, you will need to obtain permission directly from the copyright holder. To view a copy of this licence, visit http://creativecommons.org/licenses/by$\mathrm{nc} / 4.0 /$.

\section{REFERENCES}

1. Kurmani S, Squire I. Acute heart failure: definition, classification and epidemiology. Curr Heart Fail Rep. 2017;14(5):385-92.

2. Tanaka TD, Sawano M, Ramani R, Friedman M, Kohsaka S. Acute heart failure management in the USA and Japan: overview of practice patterns and review of evidence. ESC Heart Fail. 2018;5(5): 931-47.

3. Tsutsui $\mathrm{H}$, Isobe $\mathrm{M}$, Ito $\mathrm{H}$, Ito $\mathrm{H}$, Okumura $\mathrm{K}$, Ono M, et al. JCS 2017/JHFS 2017 guideline on diagnosis and treatment of acute and chronic heart failuredigest version. Circ J. 2019;83(10):2084-184.

4. Gheorghiade M, Mebazaa A. Introduction to acute heart failure syndromes. Am J Cardiol. 2005;96(6A): $1 \mathrm{G}-4 \mathrm{G}$.

5. Gheorghiade M, Mebazaa A. The challenge of acute heart failure syndromes. Am J Cardiol. 2005;96(6A): 86G-G89.

6. Valente MA, Voors AA. Renal effects of vasodilators in acute heart failure. World J Cardiovasc Dis. 2013;3(02):8.

7. Ambrosy AP, Fonarow GC, Butler J, Chioncel O, Greene SJ, Vaduganathan $\mathrm{M}$, et al. The global health and economic burden of hospitalizations for heart failure: lessons learned from hospitalized heart failure registries. J Am Coll Cardiol. 2014;63(12):1123-33.

8. Blair JE, Zannad F, Konstam MA, Cook T, Traver B, Burnett JC Jr, et al. Continental differences in clinical characteristics, management, and outcomes in patients hospitalized with worsening heart failure results from the EVEREST (Efficacy of Vasopressin Antagonism in Heart Failure: Outcome Study with Tolvaptan) program. J Am Coll Cardiol. 2008;52(20):1640-8.

9. Mentz RJ, Cotter G, Cleland JG, Stevens SR, Chiswell $\mathrm{K}$, Davison BA, et al. International differences in clinical characteristics, management, and outcomes in acute heart failure patients: better shortterm outcomes in patients enrolled in Eastern Europe and Russia in the PROTECT trial. Eur J Heart Fail. 2014;16(6):614-24.

10. Yaku H, Ozasa N, Morimoto T, Inuzuka Y, Tamaki Y, Yamamoto E, et al. Demographics, management, and in-hospital outcome of hospitalized acute heart failure syndrome patients in contemporary real clinical practice in Japan-observations from the prospective, multicenter Kyoto Congestive Heart Failure (KCHF) Registry. Circ J. 2018;82(11):2811-9.

11. Shiraishi Y, Kohsaka S, Sato N, Takano T, Kitai T, Yoshikawa T, et al. 9-Year trend in the management of acute heart failure in japan: a report from the national consortium of acute heart failure registries. J Am Heart Assoc. 2018;7(18):e008687.

12. Yancy CW, Lopatin M, Stevenson LW, De Marco T, Fonarow GC, Committee ASA, et al. Clinical presentation, management, and in-hospital outcomes of patients admitted with acute decompensated heart failure with preserved systolic function: a report from the Acute Decompensated Heart Failure National Registry (ADHERE) Database. J Am Coll Cardiol. 2006;47(1):76-84.

13. Mizuno A, Iguchi H, Sawada Y, Hurley M, Nomura $\mathrm{H}$, Hayashi $\mathrm{K}$, et al. The impact of carperitide usage on the cost of hospitalization and outcome in patients with acute heart failure: high value care vs. low value care campaign in Japan. Int J Cardiol. 2017;241:243-8.

14. Yasuda S, Nakao K, Nishimura K, Miyamoto Y, Sumita Y, Shishido T, et al. The current status of cardiovascular medicine in Japan-analysis of a large number of health records from a Nationwide Claim-Based Database. JROAD-DPC Circ J. 2016;80(11):2327-35.

15. Nagai T, Iwakami N, Nakai M, Nishimura K, Sumita Y, Mizuno A, et al. Effect of intravenous carperitide versus nitrates as first-line vasodilators on in-hospital outcomes in hospitalized patients with acute heart failure: Insight from a nationwide claimbased database. Int J Cardiol. 2019;280:104-9.

16. Kanaoka K, Okayama S, Nakai M, Sumita Y, Onoue $\mathrm{K}$, Soeda $\mathrm{T}$, et al. Number of cardiologists per cardiovascular beds and in-hospital mortality for acute 
heart failure: a nationwide study in Japan. J Am Heart Assoc. 2019;8(18):e012282.

17. Konishi M, Matsuzawa Y, Ebina T, Kosuge M, Gohbara M, Nishimura K, et al. Impact of population density on mortality in patients hospitalized for heart failure-JROAD-DPC registry analysis. J Cardiol. 2020;75(4):447-53.

18. Shiga T, Suzuki A, Haruta S, Mori F, Ota Y, Yagi M, et al. Clinical characteristics of hospitalized heart failure patients with preserved, mid-range, and reduced ejection fractions in Japan. ESC Heart Fail. 2019;6(3):475-86.

19. JCS Joint Working Group. Guidelines for rehabilitation in patients with cardiovascular disease (JCS 2012). Circ J. 2014; 78(8):2022-93.

20. Medical Vision Data. https://www.mdv.co.jp/ solution/pharmaceutical/english/. Accessed 7 Nov 2019.

21. Deyo RA, Cherkin DC, Ciol MA. Adapting a clinical comorbidity index for use with ICD-9-CM administrative databases. J Clin Epidemiol. 1992;45(6): 613-9.

22. Matsue Y, Damman K, Voors AA, Kagiyama N, Yamaguchi T, Kuroda S, et al. Time-to-furosemide treatment and mortality in patients hospitalized with acute heart failure. J Am Coll Cardiol. 2017;69(25):3042-51.

23. Aizawa H, Imai S, Fushimi K. Factors associated with 30-day readmission of patients with heart failure from a Japanese administrative database. BMC Cardiovasc Disord. 2015;15:134.

24. Sasaki N, Kunisawa S, Ikai H, Imanaka Y. Differences between determinants of in-hospital mortality and hospitalisation costs for patients with acute heart failure: a nationwide observational study from Japan. BMJ Open. 2017;7(3):e013753.

25. Sato N, Kajimoto K, Keida T, Mizuno M, Minami Y, Yumino D, et al. Clinical features and outcome in hospitalized heart failure in Japan (from the ATTEND Registry). Circ J. 2013;77(4):944-51.

26. Greene S, Triana T, Ionescu-Ittu R, Burne R, Guérin A, Borentain $M$, et al. In-hospital therapy for heart failure with reduced ejection fraction in the United States. JACC Heart Fail. 2020;8(11):943-53.

27. Sakamoto H, Rahman M, Nomura S, Okamoto E, Koike S, Yasunaga $H$, et al. Japan health system review. World Health Organization. Regional Office for South-East Asia. License: CC BY-NC-SA 3.0 IGO. Health Systems in Transition Vol. 8 No. 12018. https://apps.who.int/iris/handle/10665/259941.

28. Goto Y. Current state of cardiac rehabilitation in Japan. Prog Cardiovasc Dis. 2014;56(5):557-62.

29. Nagai T, Sundaram V, Rothnie K, Quint JK, Shoaib A, Shiraishi Y, et al. Mortality after admission for heart failure in the UK compared with Japan. Open Heart. 2018;5(2):e000811.

30. Hashimoto H, Ikegami N, Shibuya K, Izumida N, Noguchi $H$, Yasunaga $H$, et al. Cost containment and quality of care in Japan: is there a trade-off? Lancet. 2011;378(9797):1174-82.

31. McKee SP, Leslie SJ, LeMaitre JP, Webb DJ, Denvir MA. Management of chronic heart failure due to systolic left ventricular dysfunction by cardiologist and non-cardiologist physicians. Eur J Heart Fail. 2003;5(4):549-55.

32. Hoorn C, Crijns H, Dierick-van Daele A, Dekker L. Review on factors influencing physician guideline adherence in cardiology. Cardiol Rev. 2019;27(2): 80-6.

33. Matsue Y, Kagiyama N, Yoshida K, Kume T, Okura $\mathrm{H}$, Suzuki M, et al. Carperitide is associated with increased in-hospital mortality in acute heart failure: a propensity score-matched analysis. J Card Fail. 2015;21(11):859-64.

34. Hata N, Seino Y, Tsutamoto T, Hiramitsu S, Kaneko $\mathrm{N}$, Yoshikawa T, et al. Effects of carperitide on the long-term prognosis of patients with acute decompensated chronic heart failure: the PROTECT multicenter randomized controlled study. Circ J. 2008;72(11):1787-93.

35. The Japanese Circulation Society/The Japanese Heart Failure Society: Statement of proper use of vasopressin V2 receptor antagonist (Japanese). 2013. $\quad$ htttp://asas.or.jp/jhfs/pdf/20131021_ statement.pdf (asas.or.jp).

36. Shirakabe A, Hata N, Yamamoto M, Kobayashi N, Shinada T, Tomita K, et al. Immediate administration of tolvaptan prevents the exacerbation of acute kidney injury and improves the mid-term prognosis of patients with severely decompensated acute heart failure. Circ J. 2014;78(4):911-21. 